\title{
Monotone Comparative Statics for Models of Politics
}

\author{
Scott Ashworth Princeton University \\ Ethan Bueno de Mesquita Washington University
}

\begin{abstract}
We elucidate a powerful yet simple method for deriving comparative statics conclusions for a wide variety of models: Monotone Comparative Statics (Milgrom and Shannon 1994). Monotone comparative static methods allow researchers to extract robust, substantive empirical implications from formal models that can be tested using ordinal data and simple nonparametric tests. When these methods apply, they can replace a diverse range of more technically difficult mathematics (facilitating richer, more realistic models), assumptions that are hard to understand or justify substantively (highlighting the political intuitions underlying a model's results), and a complicated set of methods for extracting implications from models. We present an accessible introduction to the central monotone comparative statics results and a series of practical tools for using these techniques in applied models (with reference to original sources, when relevant). Throughout we demonstrate the techniques with examples drawn from political science.
\end{abstract}

$\mathrm{F}$ ormal theorists typically base the testable predictions of their models on comparative statics-the analysis of how changes in the parameters of a model affect the model's solution. For instance, within a legislative-institutional equilibrium a researcher might ask what happens to the cohesiveness of party votes when the competitiveness of elections increases. Similarly, in a model of judicial politics an analyst might want to predict how the level of deference to precedent changes with the independence of the judiciary. Despite the centrality of this approach, Cameron and Morton (2002) point out that no textbook for political scientists discusses any general techniques for finding comparative statics. In this article we elucidate a powerful yet simple method for deriving comparative statics conclusions for a wide variety of models. This approach is known as Monotone Comparative Statics (Milgrom and Shannon 1994).

The tools associated with monotone comparative statics offer several advantages over other techniques, making them invaluable to scholars interested in solving or testing applied formal models. Indeed, as will become clear throughout this article, an understanding of the basics of monotone comparative statics will allow applied researchers to solve and deduce testable implications from a wide array of substantive models that would otherwise either be intractable or require knowledge of a much larger, and more technically difficult, set of techniques.

We want to be clear that the purpose of this article is not to make a contribution to the theory of monotone comparative statics itself. The original sources for all theorems are cited in the text. Our purpose is to demonstrate to political scientists, heretofore largely unfamiliar with monotone comparative statics, how these tools can be employed in models of politics.

When the conditions for monotone comparative statics are met, the analysis of a model is significantly simplified. The monotone comparative statics approach identifies simple sufficient conditions for observable implications. The simplicity of these conditions makes them easy to verify. When the analysis of comparative statics is simplified to this degree, the researcher is able to solve richer, more general, and more empirically relevant models. Thus, this approach frees applied formal theorists to think about models that otherwise would have been intractable.

Another advantage is that, when monotone comparative statics are present in a model, they facilitate the development of implications of theoretical models that are directly relevant for empirical testing. First, monotone comparative static predictions are robust against a large

Scott Ashworth is assistant professor of politics, Princeton University, 130 Corwin Hall, Princeton NJ 08544 (sashwort@princeton.edu). Ethan Bueno de Mesquita is assistant professor of political science, Campus Box 1063, Washington University, One Brookings Dr., St. Louis MO 63130 (ebuenode@artsci.wustl.edu).

We thank Gary King for encouraging us to start this project and for helpful comments on an early version. We have also benefited from comments by Bruce Bueno de Mesquita, Amanda Friedenberg, Dan Ho, Andrew Martin, and an anonymous referee.

American Journal of Political Science, Vol. 50, No. 1, January 2006, Pp. 214-231 
class of misspecifications of the theoretical model. Since formal theorists generally believe that their models are simplifications, rather than complete descriptions of the world, if one wants to test a theoretical prediction it is important to know that one's hypotheses would not be fundamentally undermined by model misspecification. Second, monotone comparative statics often generate predictions that do not depend on the full specification of the model but only on a key monotonicity relationship. As such, this approach generates empirical predictions that can be tested using purely ordinal information and nonparametric methods whose consistency does not depend on the correctness of the full range of assumptions underlying the theoretical model, unlike many common statistical procedures employed by quantitative empirical researchers.

Finally, the monotone comparative statics approach allows a modeler to identify a class of critical substantive assumptions that drive the predictions of his or her model. Other approaches to comparative statics (such as choosing functional forms or employing the implicit function theorem, which we discuss later) often rely on technical conditions, such as differentiability, to show how a variable of interest changes with a parameter. In general, differentiability does not have any political meaning. As such, if the researcher wants to deduce observable implications from a model, he or she is forced to make purely technical assumptions without substantive justification. When the monotone comparative statics approach applies, on the other hand, the researcher can easily identify the key assumptions that must hold in order for his or her comparative static claims to be valid. This allows extraneous technical assumptions to be dropped. Moreover, the assumptions that remain are often (though not always) in a form that can be evaluated through qualitative research and expertise. For instance, we demonstrate that in a model of appeasement, the level of appeasement will be decreasing in the level of military expenditures only if military expenditures and the underlying state of military technology are complements. That is, we make a substantive prediction (appeasement is decreasing in military expenditures) and show that it will be true in the model as long as a substantive assumption (better military technology increases the efficacy of military spending) that can be evaluated qualitatively holds. An expert can decide whether this is a reasonable assumption and, therefore, whether or not to credit the model's predictions. We will develop this idea in considerable detail, employing a variety of examples, as the article proceeds.

Of course, comparative statics are interesting even when they are not monotone. Thus other methods for comparative statics retain their usefulness, since they ap- ply in some situations where the techniques discussed here do not. Thus we do not see monotone comparative statics as a panacea that solves all comparative static problems. Rather, these results identify an important class of models in which the comparative statics properties are easily determined.

The article is organized as follows. We start with a simple example to provide intuitions. This example is followed by an introduction to the central monotone comparative static results and practical tools for using these techniques in applied models. These sections should be accessible, with some work, to applied researchers who are familiar with basic formal theoretic tools. Of necessity, as we continue to more advanced topics in later sections - including games with strategic complementarity and monotone comparative statics under uncertainty - the presentation becomes somewhat more technical. This material will still be accessible to scholars whose research agenda is not pure formal theory, but will require a considerably greater investment of time. Our hope is that this organization will make the article useful to a variety of scholars, from those who want only to learn the basic techniques in order to deduce and test robust implications from simple models to more advanced formal theorists seeking an introduction to a set of tools they may not have previously encountered. Throughout we demonstrate the techniques with examples drawn from political science. Although this article is self-contained, we also provide references to some of the more advanced theoretical literature on monotone comparative statics for those who are interested in further reading. We conclude with a discussion of the implications of monotone comparative statics for quantitative empirical research.

\section{Motivating Example: The Appeasement Problem}

Powell (2002) studies the following problem, which we will call the appeasement problem. (See Fearon (1995) for related models.) Two states must divide some territory. There is a status quo division, but one state (call it $D$ ) is dissatisfied with that status quo. The other state (call it $S$ ) is satisfied with the status quo division.

$S$ gets one chance to try to appease $D$ by offering it some of the disputed territory. Let $x$ be the fraction of $S$ 's territory that it offers. $S$ is uncertain about how dissatisfied $D$ is. $S$ believes that $D$ will accept an offer of $x$ with probability $p(x)$. Assume that $p(x)$ is strictly increasing, so $x>x^{\prime}$ implies that $p(x)>p\left(x^{\prime}\right)$. The better the offer, the more likely $D$ is to accept it. If $D$ accepts 
the offer, then war is averted and $S$ is left with $1-x$ of its territory. If $D$ rejects the offer, then there is a war.

$S$ believes that it will win a war with probability $q$. Thus $q$ can be thought of as $S$ 's relative military strength. If $S$ wins a war, then $S$ keeps all of its territory. If $S$ loses the war, it ends up with none of the disputed territory. Given all of this, $S$ will choose the offer of appeasement, $x$, to solve the following maximization problem:

$$
\max _{x}(1-x) p(x)+q(1-p(x)) .
$$

The comparative static question of interest in this model is how the level of appeasement, $x$, changes with $S$ 's perception of its military strength, $q$. Does $S$ offer more or less when it perceives itself to be strong? This is a classic comparative static question, asking how an output of the model (the level of appeasement) changes with a parameter of the model (the probability that $S$ wins a war with $D)$, holding all else constant.

One way to find the comparative statics for this problem is to choose a specific functional form for $p$ and calculate the solution explicitly. For example, suppose $p(x)=x$. This means that $S$ believes that if it offers onehalf of the disputed territory, then $D$ accepts that offer with probability one-half and likewise for all offers between 0 and 1 . Under this assumption, $S$ 's maximization problem can be rewritten as follows:

$$
\max _{x}(1-x) x+q(1-x) .
$$

We can solve this using simple calculus. The first-order condition is:

$$
1-2 x-q=0,
$$

so the solution is

$$
x^{*}(q)=\frac{1-q}{2},
$$

where $x^{*}(q)$ represents state $S$ 's optimal choice of appeasement as a function of $S$ 's perceived military strength. Thus, for any given probability that $S$ would win a war $(q)$ we know precisely what offer $S$ will make to try to appease $D$. We can find comparative statics by examining how this equilibrium offer $\left(x^{*}(q)\right)$ changes when $q$ changes. Differentiating $x^{*}(q)$ with respect to $q$ yields:

$$
\frac{d x^{*}}{d q}(q)=-\frac{1}{2}<0
$$

Not surprisingly, the optimal offer is decreasing in $q$. The stronger $S$ is militarily, the less willing $S$ is to appease $D$.

While this final conclusion seems intuitive, the preceding derivation relied on the functional form of $p$, the probability that $D$ accepts an offer. Without this functional form $(p(x)=x)$ the analysis would not have simplified in the nice way that it did and so we would not have had an explicit solution for the optimal offer. ${ }^{1}$ But the functional form was not chosen because of some compelling substantive political argument that the probability of an offer being accepted is linear in the offer. Rather, it was chosen because it simplified the algebra. This is troublesome for at least two reasons. First, if the conclusion, rather than just the derivation, relies on the functional form, then the conclusion is suspect unless the functional form can be substantively justified. Second, this approach makes it hard to generalize or add nuance to the results, since to solve a richer model we would still have to find functional forms that lead to explicit solutions, a process which gets harder and harder as the model becomes more realistic.

To see this second point more clearly, consider an intuitively appealing extension of the appeasement problem. One might like to model $S$ as having two decisions to make rather than one. For example, suppose that $S$ 's perceived military strength is actually the result of both some preexisting military capacity and the level of military buildup during the negotiations. Write this probability as $q(z, \theta)$, where $z$ is preparation for fighting and $\theta$ is preexisting military capacity. It is natural to assume that $S^{\prime}$ s military strength is increasing in both military buildup $(\partial q / \partial z>0)$ and in preexisting capacity $(\partial q / \partial \theta>0)$. Further, a military buildup is costly. We represent this cost with a function $c(z)$. Finally, it seems sensible to think that the likelihood $D$ accepts an offer of appeasement depends on both the offer $(x)$ and $S$ 's military buildup during the crisis, so the probability of acceptance is some function $p(x, z)$.

To solve this richer, more realistic model, we have to find $S$ 's optimal offer by analyzing the following maximization problem:

$$
\max _{x, z}(1-x) p(x, z)+q(z, \theta)(1-p(x, z))-c(z) .
$$

It would be nice to be able to analyze this without making strong and substantively unjustified functional form assumptions. Indeed, choosing a functional form for $p(x$, $z$ ) that leads to a simple explicit solution is no longer a simple task. While it may have been quite easy to select the functional form $p(x)=x$ in the first example, what is the obvious choice for the functional form of $p(x, z)$ ?

Motivated by the desire to solve models of this sort we now turn to the theory of monotone comparative statics. We begin by developing techniques that are useful for solving general decision theoretic problems such as the

\footnotetext{
${ }^{1}$ An explicit solution, as opposed to an implicit solution, is one in which the variable being solved for appears on its own on one side of an equal sign.
} 
one above. We then extend these techniques for use in game theoretic settings. ${ }^{2}$

\section{The Theory of Monotone Comparative Statics}

In developing the theory, we start with the simplest case: a one-dimensional decision. We will develop this rigorously and then show intuitively how the techniques extend to more general cases. This will allow us to solve our motivating example above, as well as a host of other political science models.

Before proceeding, one technical point is necessary. When developing more general models in the way that we advocate, one is not always assured of having unique solutions. For ease of exposition, while developing the theory we assume unique solutions. However, this assumption is not necessary in order to reach comparative static conclusions. Indeed, one of the strengths of the monotone comparative statics approach is that it allows one to make comparative static statements even when there are multiple equilibria. However, it is easier to explain the basics when there is only one solution. We return to the issue of multiplicity later in this section.

We will study comparative statics in the context of a general optimization problem:

$$
\max _{a \in A} f(a, \theta),
$$

where $\theta$ is a parameter and $a$ is a choice variable. The function to be maximized is sometimes called the objective function. We assume that $a \in A \subset \mathbb{R}$ and $\theta \in \Theta \subset \mathbb{R}$. We want to know how the optimal choice, $a^{*}$, changes as $\theta$ changes.

This general optimization problem can represent a host of political decisions. As in our opening example, it might represent the optimal level of appeasement given a state's military strength. In this case, $a^{*}$ corresponds to the optimal offer ( $x^{*}$ in our opening example) and $\theta$ corresponds to $S$ 's military strength ( $q$ in our opening example). Another political problem this optimization problem could represent is the decision over what tax

\footnotetext{
${ }^{2}$ The distinction between decision and game-theoretic problems is that in decision-theoretic problems we consider the choices of only one actor, holding other actors actions constant (or assuming they are nonstrategic). In game theoretic problems we have multiple actors, all of whom are strategic. For example, to make the appeasement problem game theoretic, we would model the state $D$ as a strategic actor and have the probability that $D$ rejects an offer be an optimal choice within an equilibrium. We will address such problems, but expositionally it is easier to begin with decision theory.
}

policy a legislator supports ( $a$ ) given the percentage of people in her district living below the poverty line $(\theta)$. One can come up with many further such examples.

Consider two possible choices, $a$ and $a^{\prime}$, where $a>$ $a^{\prime}$. If the problem is choice of tax policy, then $a$ would be a higher tax rate than $a^{\prime}$. In order to think about monotone comparative statics, we will need to study the function $f(a, \cdot)-f\left(a^{\prime}, \cdot\right)$, which is called the incremental return. The incremental return is a measure of the benefit (or loss if it is negative) that our decision maker realizes by moving from the lower choice $\left(a^{\prime}\right)$ to the higher one $(a)$. Clearly, for any given level of the parameter $\theta$, the decision maker prefers $a$ to $a^{\prime}$ if and only if $f(a, \theta)-f\left(a^{\prime}, \theta\right)>$ 0 .

In order to derive monotone comparative statics, we will study a special property of the incremental return known as the single-crossing condition. We will show that when the single-crossing property holds, then powerful comparative statics follow.

The single-crossing condition requires that the incremental return change sign only once as the parameter $\theta$ increases. That is, suppose that a decision maker prefers policy $a^{\prime}$ to policy $a$ when $\theta$ is small. This means that, for a sufficiently small $\theta$ (call it $\theta^{\prime}$ ), the incremental return is negative. If, for some new $\theta>\theta^{\prime}$ the decision maker would prefer policy $a$ to policy $a^{\prime}$ (so that the incremental return is positive for this new parameter value $\theta$ ), then all decision makers with parameter value greater than $\theta$ must also prefer $a$ to $a^{\prime}$. Otherwise, the incremental return would change signs more than once and, therefore, would not satisfy the single-crossing condition. The single-crossing property is illustrated in Figure 1.

Definition 1. The function $f$ satisfies the single-crossing condition if for all $a>a^{\prime}$ and $\theta>\theta^{\prime}, f\left(a, \theta^{\prime}\right)-f\left(a^{\prime}, \theta^{\prime}\right) \geq$ 0 implies $f(a, \theta)-f\left(a^{\prime}, \theta\right) \geq 0$, and $f\left(a, \theta^{\prime}\right)-f\left(a^{\prime}, \theta^{\prime}\right)>$ 0 implies $f(a, \theta)-f\left(a^{\prime}, \theta\right)>0$.

At first glance, the single-crossing condition may seem hopelessly technical, belying our earlier promise to ground comparative static analysis in easy and intuitive assumptions. However, it is our intention to show that the single-crossing condition is, in fact, both easy and intuitive. We will start with why it is intuitive. Then, after developing a little more theory, we will show how checking for the existence of the single-crossing condition is often quite easy as well. First, however, in what sense is the single-crossing condition intuitive?

Consider the appeasement problem mentioned above. Imagine that there are two appeasement offers, a large appeasement or a small appeasement. Suppose that 


\section{Figure 1 Objective Functions (Left Panel) and Their Incremental Return (Right Panel) Satisfying the Single Crossing Property}

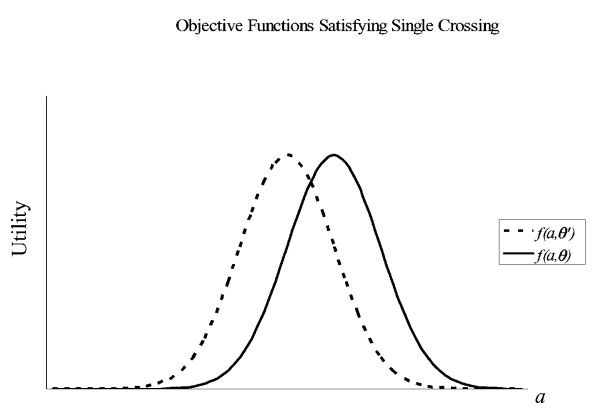

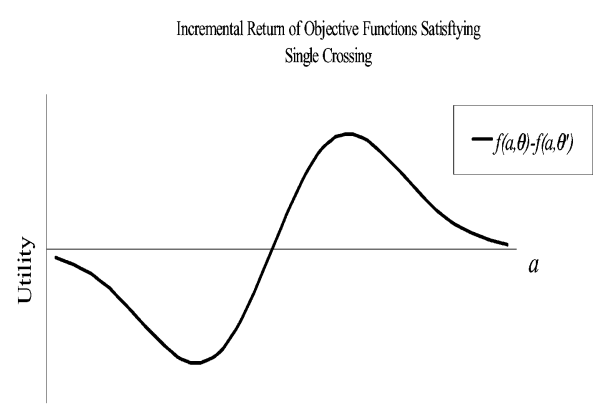

a state with relative strength $q$ prefers the large appeasement offer. Then the single-crossing condition requires that all weaker states also prefer the larger appeasement offer. The intuition underlying the single-crossing condition is also now clear: if a moderately strong state prefers the large appeasement offer, then it reveals a preference for a large concession and low probability of war to a small concession and a high probability of war. A weaker state has a lower expected payoff from war, so it has even more reason to prefer the combination of a large concession and low probability of war. The power of the monotone comparative statics approach is that comparative static results hold as long as we are comfortable making assumptions consistent with intuitive statements such as the previous sentences.

For another example, consider the tax problem mentioned above. There, the choice variable represents a tax rate and the parameter $\theta$ represents the percentage of people in a district who live below the poverty line. So, consider two possible choices of a tax rate, a high level of tax, $a$, and a low level of tax, $a^{\prime}$. Suppose, further, that a legislator with fraction $\theta^{\prime}$ of her constituents below the poverty line prefers the larger tax, $a$. Then, the single-crossing condition holds only if all other legislators from districts that are even poorer will prefer the higher tax rate to the lower tax rate (they will also have a positive incremental return). ${ }^{3}$

Now that we have seen that the single-crossing property has a substantive interpretation, we present the central result of monotone comparative statics: if the single-crossing condition holds, then the optimal choice changes monotonically with the parameter. Throughout

\footnotetext{
${ }^{3}$ Readers might note a similarity between the intuition of single crossing and single peakedness. It turns out that, while the intuitions are similar, these are logically distinct properties, both of which are sufficient conditions for the existence of a median voter theorem (Gans and Smart 1996).
}

the article, theorems are presented without proof (though we always provide the technical intuition and a citation to the proof).

Theorem 1. (Milgrom and Shannon (1994)) Assume that $f$ satisfies the single-crossing property and let $a^{*}(\theta)=$ $\arg \max _{a \in A} f(a, \theta)$. Then $a^{*}$ is weakly increasing in $\theta$.

The proof of this result follows straightforwardly from the intuitive interpretation of the single-crossing condition. Consider a decision maker with type $\theta^{\prime}$ who prefers $a$ to $a^{\prime}$. Her payoff function satisfies $f(a, \theta)-$ $f\left(a^{\prime}, \theta\right)>0$, which we can rewrite as

$$
f\left(a, \theta^{\prime}\right)-f\left(a^{\prime}, \theta^{\prime}\right)>0 .
$$

If $f$ satisfies the single-crossing property, then we have

$$
f(a, \theta)-f\left(a^{\prime}, \theta\right)>0
$$

for all $\theta>\theta^{\prime}$. Thus a decision maker with type $\theta$ has $f(a$, $\theta)>f\left(a^{\prime}, \theta\right)$, which says that she also prefers $a$ to $a^{\prime}$. This argument shows that, if type $\theta^{\prime}$ chooses $a$, then every type $\theta>\theta^{\prime}$ prefers $a$ to any alternative $a^{\prime}<a$. Thus a higher type cannot choose a lower alternative.

We have, thus far, seen that the single-crossing property is intuitive and powerful. However, these alone do not make it useful. To make this concept practical, we need a way to check whether or not it holds so that we can know whether or not we have monotone comparative statics in an applied model. It turns out that there is a sufficient condition for single crossing, called increasing differences, that is easy to check in applications. We develop this next.

\section{Increasing Differences: A Sufficient Condition for Single Crossing}

We begin with a definition of increasing differences, which is illustrated in Figure 2. 


\section{FIGURE 2 Objective Functions (Left Panel) and Their Incremental Return (Right Panel) Satisfying Both the Single Crossing Property and Increasing Differences}
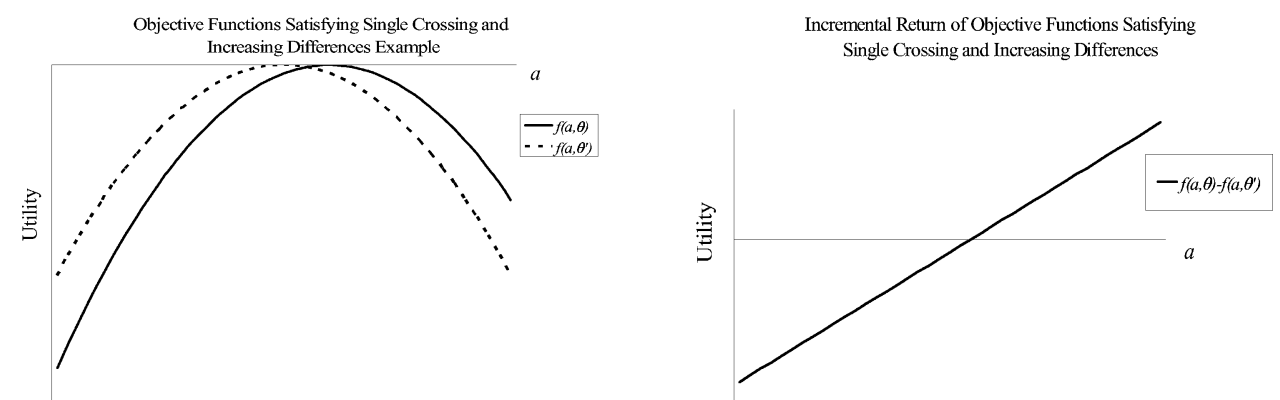

Definition 2. The function $f$ has increasing differences if the incremental return, $f(a, \cdot)-f\left(a^{\prime}, \cdot\right)$, is weakly increasing in the parameter, $\theta$.

If the incremental return is weakly increasing, then the single-crossing condition is satisfied. Why is this? Increasing differences is true if:

$$
f(a, \theta)-f\left(a^{\prime}, \theta\right) \geq f\left(a, \theta^{\prime}\right)-f\left(a^{\prime}, \theta^{\prime}\right),
$$

for all $a>a^{\prime}$ and $\theta>\theta^{\prime}$. If $f\left(a, \theta^{\prime}\right)-f\left(a^{\prime}, \theta^{\prime}\right) \geq 0$, then we have the following chain of inequalities

$$
f(a, \theta)-f\left(a^{\prime}, \theta\right) \geq f\left(a, \theta^{\prime}\right)-f\left(a^{\prime}, \theta^{\prime}\right) \geq 0,
$$

and $f$ is single crossing. Notice, however, that the converse is not true. An incremental return can satisfy single crossing without having increasing differences, as illustrated in Figure 1.

Increasing differences is a generalization of the classical idea of complementarity-an increase in the parameter increases the marginal benefit of an increase in the choice variable. Another way of interpreting this is that, the higher the value of the parameter, the more efficacious is an increase in the choice variable. For example, in the appeasement problem, if state strength and offers of appeasement are complements, then the stronger the state is the more likely a small increase in the appeasement offer is to lead to acceptance. Under convexity and smoothness assumptions, the optimal choice in a formal model sets marginal benefits equal to marginal costs. When the parameter and choice variable are complements, an increase in the parameter increases the marginal benefits, which then increases the optimal choice. The monotonicity theorem based on increasing differences generalizes this result so that it holds without the extraneous assumptions regarding convexity and smoothness. ${ }^{4}$

\footnotetext{
${ }^{4}$ We discuss a special case of this, discrete choice, below.
}

If the objective function $(f)$ is twice continuously differentiable, then increasing difference becomes trivial to verify. To see this, write the definition of increasing differences as

$$
\left[f(a, \theta)-f\left(a^{\prime}, \theta\right)\right]-\left[f\left(a, \theta^{\prime}\right)-f\left(a^{\prime}, \theta^{\prime}\right)\right] \geq 0,
$$

for all $a>a^{\prime}$ and $\theta>\theta^{\prime}$. Notice that if $f$ is twice continuously differentiable, then for small changes in a, the first term in square brackets is approximated by $\frac{\partial f(a, \theta)}{\partial a}\left(a-a^{\prime}\right)$ and the second term by $\frac{\partial f\left(a, \theta^{\prime}\right)}{\partial a}\left(a-a^{\prime}\right)$. Since $\left(a-a^{\prime}\right)$ is positive, increasing differences can be rewritten as

$$
\frac{\partial f(a, \theta)}{\partial a}-\frac{\partial f\left(a, \theta^{\prime}\right)}{\partial a} \geq 0 .
$$

Now notice that this condition says that the derivative of the objective function with respect to $a$ must be weakly increasing in the parameter. That can be written as follows:

$$
\frac{\partial^{2} f(a, \theta)}{\partial a \partial \theta} \geq 0 \text {. }
$$

This condition is easy to check. In particular, we can confirm that, the optimal choice has monotone comparative statics with respect to some parameter by taking a simple cross partial derivative. Thus, the single-crossing property is indeed powerful, intuitive, and useful.

\section{Discrete Choice and Multiple Optima}

It is worth noting that monotone comparative static results do not depend on differentiability. We used differentiability to verify the single-crossing property in a particularly easy way, but the monotonicity theorem was stated entirely in terms of the single-crossing property. This is important because in some applied settings differentiability is not a good assumption. Consider a couple of examples. In a model of parliamentary coalition 
formation, the actors might be extreme-leftist, left-center, centrist, right-center, and extreme-rightist parties. The centrist party might have to choose which other parties to bring into a governing coalition. There is no sensible way to model this decision continuously-the choice is inherently discrete. Similarly, in a model of conflict and appeasement (which we discuss in more detail later) the land under dispute might have religious significance making partition infeasible.

In such discrete choice situations, we can still reach comparative static conclusions using monotone comparative static techniques. This is because, unlike other techniques (such as the implicit function theorem), monotone comparative statics do not depend on differentiability in any way. We do not, for instance, need to exploit a first-order condition to do comparative statics, we simply need to determine whether the incremental return is single crossing.

Moreover, in discrete choice situations, the objective function may sometimes be differentiable in the parameter $(\theta)$ even though it is not differentiable in the choice variable $(a)$. For instance, in an appeasement setting the land might not be infinitely divisible. If the parameter is something continuous, such as relative military power, then it is reasonable to assume differentiability in the parameter. In this case, we can check whether the incremental return is weakly increasing using calculus. Recall that the incremental return is $f(a, \theta)-f\left(a^{\prime}, \theta\right)$. The incremental return is weakly increasing if, for all $\theta \in \Theta$ and $a$, $a^{\prime} \in A$ with $a>a^{\prime}$ :

$$
\frac{\partial}{\partial \theta}\left[f(a, \theta)-f\left(a^{\prime}, \theta\right)\right] \geq 0 .
$$

This condition is particularly easy to verify in a model with only two possible actions. In this case, monotone comparative statics imply a cutpoint decision rule; there is a parameter value $\theta^{*}$ such that the decision maker chooses the smaller action if $\theta<\theta^{*}$ and she chooses the larger action if $\theta>\theta^{*}$.

Thus far, we have presented the results assuming that a unique solution to the decision problem exists. However, our current focus on discrete choice forces us to confront the issue of multiple optima. In the typical case of discrete choice with continuous payoff functions, the decision maker will be indifferent between two choices at the parameter value where she switches from one choice to the other. For example, in the cutpoint case, the decision maker is indifferent between the two actions if $\theta=\theta^{*}$. Thus, at that point there are multiple optima. ${ }^{5}$ An

${ }^{5}$ This problem is not limited to discrete choice but also arises whenever the global maximum of a nonquasiconcave objective changes from one local maximum to another. advantage of the monotone comparative statics approach is that it allows us to draw comparative static conclusions even in these cases.

This appearance of multiple optima raises an important question - what does monotonicity mean for sets of solutions? The literature offers several answers to this question; we use the simplest one. Say that a set of solutions $S_{1}$ is greater than another set of solutions $S_{2}$ if the greatest element of $S_{1}$ is larger than the greatest element of $S_{2}$ and the least element of $S_{1}$ is larger than the least element of $S_{2}$. (Notice that this definition of monotonicity reduces to the standard order on real numbers when there is a unique optimum.) All theorems we have stated (and will state throughout) hold exactly as stated if we substitute this concept of increasing sets for the usual concept of monotonicity (Milgrom and Shannon 1994).

\section{An Application to the Appeasement Problem}

Now that we have presented the basic theory, let's put it to work in our motivating example. In our opening treatment of the appeasement problem we chose a specific functional form for $p$, the probability that the offer is accepted, in order to derive comparative statics from an explicit solution. Using monotone comparative static techniques we can derive these comparative statics without assuming a functional form, or even concavity of the objective function.

Consider the one-dimensional problem:

$$
\max _{x}(1-x) p(x)+q(1-p(x)) .
$$

Assume that $p$ is differentiable and recall that $p$ is increasing, that is the probability that an offer is accepted is increasing in the size of that offer. We want to determine whether the optimal offer $x^{*}$ is decreasing in the decisionmaking country's military strength $(q)$. The monotonicity results imply that this will be true if the cross partial derivative of the objective function with respect to $x$ and $q$ is negative. To check this we first differentiate with respect to $q$ to get:

$$
\frac{\partial}{\partial q} f(x, q)=(1-p(x))
$$

and then with respect to $x$ to get:

$$
\frac{\partial^{2}}{\partial x \partial q} f(x, q)=-p^{\prime}(x) \text {. }
$$

Since $p$ is increasing, $p^{\prime}(x)$ is positive, which implies that the cross-partial derivative is negative. It seems that this will stymie our comparative statics results, since the monotonicity theorem applies to objective functions with 
positive cross-partial derivatives. However, we can solve the problem using a trick called the "method of reordering." Define the parameter $\rho=-q$. Then we can rewrite the objective function as

$$
(1-x) p(x)-\rho(1-p(x)) .
$$

The cross-partial of this function is

$$
\frac{\partial^{2}}{\partial x \partial \rho} f(x, q)=p^{\prime}(x)>0 .
$$

Thus the monotonicity theorem implies that $x$ is weakly increasing in $\rho$, which means that $x$ is weakly decreasing in $q$.

Substantively, this says that, so long as more generous offers are more likely to be accepted, states with stronger chances in war will offer less territory to a dissatisfied bargaining partner. Importantly, the result depends only on the substantive claim that better offers are more likely to be accepted. We did not need the additional substantive restriction that the objective function be globally concave. ${ }^{6}$ Thus, in addition to being easy to check, the monotone comparative statics approach clarifies which assumptions drive the results.

As discussed above, if we do not assume differentiability we can still make comparative static statements. This is important substantively because one might want to analyze the appeasement problem in a case where the territory is not perfectly divisible. For example, suppose that part of the territory has important religious significance for influential groups in each state and that consequently divided control of the religious site is unacceptable to these groups. In this case, the set of feasible offers in not convex and the objective function cannot be everywhere differentiable. However, we can still say that the optimal offer is weakly decreasing in military strength since the weak comparative static conclusion depends only on increasing differences, which does not imply convexity, continuity, or differentiability.

\section{Multidimensional Decision Problems}

So far we have studied the simplest possible decision problem: one actor making one choice. We now generalize the results to show how they apply when a single decision maker has to make multiple choices and the state of the world is described by multiple parameters.

In order to address the multidimensional problem we need to extend the notion of complementarity discussed

${ }^{6}$ Global concavity follows from the restriction $\frac{p^{\prime \prime}(x)}{p^{\prime}(x)}>-1$. Convexity of $p$ is sufficient, but not necessary, for this restriction. above to a multidimensional setting. Formally characterizing the analog to the single-crossing condition for a multidimensional monotonicity theorem would require a lengthy technical tangent from our main theme. ${ }^{7}$ Instead, we will simply extend the earlier concept of increasing differences to show sufficient conditions for monotone comparative statics in a multidimensional problem. This is the essential tool for applied work.

In the one-dimensional problem we assumed that the action $a$ was in $\mathbb{R}$. Since this is now a multidimensional problem, the decision maker is choosing a vector $\mathbf{a}=$ $\left(a_{1}, a_{2}, \ldots, a_{n}\right)$. Thus, we assume that the action is $\mathbf{a} \in$ $A \subset \mathbb{R}^{n}$. Similarly, before we had a single parameter $\theta$ that described the state of the world. Now, we assume the parameter is $\mathbf{a} \in \Theta \subset \mathbb{R}^{m}$.

The multidimensional decision problem is now written:

$$
\max _{\mathbf{a}} f(\mathbf{a}, \boldsymbol{\theta}) .
$$

The extension of the earlier concept of increasing differences that we will use is called supermodularity. Before we can define supermodularity, we need another definition.

Definition 3. If the set of alternatives, $A$, is a subset of $\mathbb{R}^{n}$ and the set of parameters $\Theta$ is a subset of $\mathbb{R}^{m}$, the set $X=$ $A \times \Theta$ is a product set if it can be represented by the Cartesian product of subsets of $\mathbb{R}{ }^{8}$

Now we can define supermodularity.

Definition 4. A function $f: A \times \Theta \rightarrow \mathbb{R}$ is supermodular if $A \times \Theta$ is a product set and f has increasing differences for all pairs of arguments of the function.

For a simple example, this definition says that if we have a twice continuously differentiable function $f\left(a_{1}\right.$, $\left.a_{2}, \theta\right)$, then it is supermodular if all of the following hold: $\frac{\partial^{2} f}{\partial a_{1} \partial a_{2}}>0, \frac{\partial^{2} f}{\partial a_{1} \partial \theta}>0, \frac{\partial^{2} f}{\partial a_{2} \partial \theta}>0$. As we add in more choice alternatives or more parameters, increasing differences must hold for each possible dyad.

${ }^{7}$ The interested reader should consult Milgrom and Shannon (1994).

${ }^{8}$ For example, the square given by $\{x, y \mid x \in[0,1], y \in[0,1]\}$ is a product of set in $\mathbb{R}^{2}$. In particular, it is the Cartesian product: $[0,1] \times[0,1]$. However, the triangle given by $\{x, y \mid x \geq 0, y \geq$ $0, x+y \leq 1\}$ is not a product sets, as it cannot be written as a Cartesian product of two intervals of $\mathbb{R}$. This restriction to product sets is important because it means that monotone comparative static techniques cannot be used for problems such as the classic consumer problem, where the consumer makes a multidimensional choice subject to a budget constraint. Other powerful comparative static techniques exist for such problems, but are beyond the scope of this article. 
This is clearly a generalization of increasing differences. If $A$ and $\Theta$ are one dimensional, then the definition is exactly the same as the earlier definition of increasing differences. Moreover, as we discussed earlier, the key fact about increasing differences (and the single crossing property) that made monotone comparative statics work was complementarity. Supermodularity simply extends the notion of complementarity saying that all choices and parameters must be complements with one another.

Given this, we can state the main result, which is that supermodularity is sufficient for monotone comparative statics. We will say that the solution to the multidimensional maximization problem (labeled $\mathbf{a}^{*}$ ) has monotone comparative statics if every component is weakly increasing in the parameters. This result is formalized in the following theorem.

Theorem 2. (Topkis (1978)) Consider the problem $\max _{\mathbf{a} \in A} f(\mathbf{a}, \boldsymbol{\theta})$ where $\boldsymbol{\theta} \in \Theta$ is a parameter. If $f$ is supermodular, then the optimal solution $\mathbf{a}^{*}$ has monotone comparative statics.

This result is based on a simple idea. An increase in a parameter $\theta$ has the direct effect of weakly increasing the optimal levels of each component of the action, since supermodularity implies increasing differences for each pair of parameter and action. Since supermodularity also implies increasing differences for each pair of actions, this direct effect induces indirect effects of further increases in the action variables. Thus, all the effects of an increase in a parameter on the optimal solution are in the same direction.

Without supermodularity, the interactions between choice variables could potentially outweigh the direct effect of the change in parameters, and then the direction of comparative statics would depend on the relative size of direct and indirect effects. For example, consider the simple example of a function $f\left(a_{1}, a_{2}, \theta\right)$. Assume that $a_{1}$ has increasing differences with $\theta$ and with $a_{2}$ but that the function is not quite supermodular because $a_{2}$ does not have increasing differences with $\theta$. Now consider the effect of an increase of $\theta$ on the optimal $a_{1}$. An increase in $\theta$ leads to a direct effect of upward pressure on the optimal $a_{1}$ because of increasing differences. However, an increase in $\theta$ can also put downward pressure on the optimal $a_{2}$ which has the indirect effect of putting downward pressure on the optimal $a_{1}$, since $a_{1}$ and $a_{2}$ have increasing differences. There is no way to sign the overall effect (that is, the net effect considering both the direct and indirect effects) of an increase in $\theta$ on $a_{1}$ without making detailed assump- tions about the functional form of $f$ (precisely what we are trying to avoid doing). Thus, for monotone comparative static claims to be true we need the full strength of the supermodularity assumption. Increasing differences of a parameter and one particular component of the choice vector is not sufficient to make monotone comparative static claims about that binary relationship.

\section{The Appeasement Problem Revisited}

As we pointed out earlier, it would be nice to be able to solve a richer, multidimensional version of the appeasement problem. For instance, Bueno de Mesquita et al. (1999) present a related model in which the decisionmaking country must choose both an offer to make and how much to invest in military preparedness. ${ }^{9}$ Using the results on multidimensional problems, we can easily handle such complications.

Assume that the utility of $S$ to war depends on both some preexisting military capacity and on the level of military buildup during the negotiations. Write this utility as $q(z, \theta)$, where $z$ is preparation for fighting and $\theta$ is military capacity. It is natural to assume that $\partial q / \partial z>0$ (more preparation increases $S$ 's chance of winning) and that $\partial q / \partial \theta>0$ (preexisting capacity makes $S$ more likely to win). Increasing military preparedness is costly, with cost function $c(z)$. Now, the state solves

$$
\max _{x, z}(1-x) p(x)+q(z, \theta)(1-p(x))-c(z) .
$$

We will see that the comparative statics of this problem depend on the cross-partial $\frac{\partial^{2} q}{\partial z \partial \theta}$. This cross-partial derivative is positive only if military preparations and military capacity are complements. When might this be true? Suppose that $\theta$ measures the state of military technology and that a given increment to spending is more productive when technology is better. Under this assumption, $z$ and $\theta$ are complements and so the crosss-partial is positive.

If $z$ and $\theta$ are complements, it is straightforward to verify that the objective is supermodular in $(-x, z, \theta)$. In particular:

$$
\begin{aligned}
& \frac{\partial^{2} f(x, z, \theta)}{\partial x \partial \theta}=-\frac{\partial q}{\partial \theta}(z, \theta) p^{\prime}(x)<0 \\
& \frac{\partial^{2} f(x, z, \theta)}{\partial x \partial z}=-\frac{\partial q}{\partial z}(z, \theta) p^{\prime}(x)<0 \\
& \frac{\partial^{2} f(x, z, \theta)}{\partial z \partial \theta}=\frac{\partial^{2} q}{\partial \theta \partial z}(z, \theta)(1-p(x))>0 .
\end{aligned}
$$

${ }^{9}$ Bueno de Mesquita et al. (1999) present a game theoretic model, but it is in the spirit of our analysis here. 
This means that we can conclude that $x^{*}$ is decreasing in $\theta$ and that $z^{*}$ is increasing in $\theta$, without ever solving explicitly for $x^{*}$. Notice that an empirical researcher attempting to evaluate this model may not observe the underlying state of military technology $(\theta)$, but likely will observe the offer $(x)$ and the level of build-up $(z)$. The key observable prediction, then, is that $x$ and $z$ will be negatively correlated.

As we mentioned at the outset, a key advantage of monotone comparative statics is that it highlights the substantive content of the assumptions, so they can be examined with qualitative empirical work. For example, we just derived the result that, if preexisting military capacity makes resources devoted to a military buildup more productive (that is, if they are complements), then ex ante stronger states will choose both less generous offers and greater buildups. The key condition used in this argument concerns the effect of preexisting capacity on the efficiency of military investment, a subject which qualitative empirical work and expert interviews can shed light on. If this assumption is false, then the comparative static predictions do not hold.

The other approach that one could use to derive comparative statics from the multidimensional appeasement model is to employ the implicit function theorem. If we assume that territory and military capacity are both perfectly divisible, that the objective function is twice continuously differentiable, and that the first-order condition characterizes the optimum, then we can use the implicit function theorem to calculate the comparative static derivatives as:

$$
\begin{aligned}
& \left(\begin{array}{c}
\frac{\partial x^{*}}{\partial \theta} \\
\frac{\partial z^{*}}{\partial \theta}
\end{array}\right)= \\
& \quad-\left(\begin{array}{rr}
-2 p_{x}+(1-x-q(z, \theta)) p_{x x} & -p_{z}-q_{z} p_{x} \\
-q_{z} p_{x} & q_{z z}(1-p)-c_{z z}
\end{array}\right)^{-1} \\
& \quad \times\left(\begin{array}{c}
-q_{\theta} p_{x} \\
q_{z \theta}(1-p(x))
\end{array}\right) .
\end{aligned}
$$

The implicit function theorem does, in the end, lead to the same comparative static predictions as the monotone comparative statics approach with differentiability. However, two significant advantages to monotone comparative statics are evident. First, for monotone methods we did not need to assume convexity or differentiability. The implicit function theorem, however, only works if the first-order condition characterizes the optimum. Thus, the implicit function theorem requires stronger as- sumptions and cannot be applied to models such as the appeasement problem when the territory is not perfectly divisible. ${ }^{10}$ Second, whenever the implicit function approach does work, the monotone comparative statics approach also works while only requiring that the modeler calculate simple cross-partial derivatives.

\section{Further Tools}

So far, we have concentrated on the role of increasing differences and supermodularity in ensuring that choice variables are weakly increasing in the parameters. In this section, we tie up a couple of loose ends in this discussion. First, we discuss an alternative sufficient condition for the single-crossing property that is useful in multiplicatively separable problems. Second, we discuss the problem of ensuring that the solution is strictly increasing in the parameters. Although the results we present hold for multidimensional problems, we simplify the discussion by restricting attention to one-dimensional choices.

\section{Log-supermodularity}

In some situations, the decision maker's objective function may not be supermodular, but we can still apply monotone comparative statics. Recall that supermodularity is a sufficient, but not necessary condition for monotone comparative statics. In certain circumstances, a useful trick makes it possible to demonstrate that a decision problem has the needed complementarities even though supermodularity of the objective function cannot be established. This trick involves taking logs. We illustrate this using an example.

Consider the following simple model of election spending. ${ }^{11}$ A candidate is running for office as a challenger and must decide how much money to devote to campaign spending $a$. The probability that the challenger wins $p(a, \theta)$ is increasing in campaign spending and decreasing in the incumbent's level of name recognition $\theta>0 .{ }^{12}$ The candidate also gains some utility from holding office, $u(a)$. This utility is a decreasing function of

\footnotetext{
${ }^{10}$ See Fearon (1995) for a discussion of the importance of models of conflict without perfect divisibility.

${ }^{11}$ This simple decision-theoretic model is related to more sophisticated models studied by Erikson and Palfrey (2000), Grossman and Helpman (1996), and Baron (1994).

${ }^{12}$ To keep the example simple, we do not model strategic behavior by other candidates, though doing so along the lines of the gametheoretic methods discussed below would only reinforce the point.
} 
the amount of resources spent on campaigning $\left(\frac{\partial u}{\partial a}<0\right)$. This assumption reflects the idea that campaign funds are raised in exchange for promises that the challenger will provide favors to interest groups if she wins, and that providing these favors uses resources that the candidate could otherwise use to pursue her own legislative goals.

The candidate's objective function is $U=p(a$, $\theta) u(a)$, and he solves the following maximization problem:

$$
\max _{a} p(a, \theta) u(a) .
$$

Unfortunately, the candidate's objective function may not be supermodular. To see this, note that

$$
\frac{\partial^{2} U}{\partial a \partial \theta}=\frac{\partial u}{\partial a} \frac{\partial p}{\partial \theta}+\frac{\partial^{2} p}{\partial a \partial \theta} u .
$$

The first term here is clearly positive, but the second term need not be. For instance, consider a stochastic voting model where the candidate believes that he will win the pivotal voter's vote only if $\theta+\epsilon<a$, where $\epsilon$ is a normally distributed, random variable. The probability of the candidate winning, then, is $p(a, \theta)=\Phi(a-\theta)$, where $\Phi$ is the cumulative density function of the standard normal distribution. The cross partial probability that the candidate wins is given by:

$$
\frac{\partial^{2} p}{\partial a \partial \theta}=\frac{\partial^{2} \Phi(a-\theta)}{\partial a \partial \theta}=-\phi^{\prime}(a-\theta),
$$

where $\phi$ is the probability density function (pdf) of the normal distribution. Since the pdf of the normal is increasing to the left of its mean and decreasing to the right of its mean, the sign of $\phi^{\prime}(a-\theta)$ changes depending on the values of $a$ and $\theta$. Thus, the cross-partial cannot be signed and so, even using the normal distribution, the objective function may not be supermodular.

Fortunately, comparative statics conclusions continue to hold when the objective function is transformed in any monotonic way. A particularly convenient such transformation is to take logarithms. Hence, to attempt to establish comparative statics of our elections model, we can study the transformed maximization problem:

$$
\max _{a}(\log p(a, \theta)+\log u(a))
$$

If this transformed problem is supermodular, then the monotonicity theorem still implies that there are monotone comparative statics. Since this transformation is so broadly useful, we give a formal definition.
Definition 5. The function $f: A \times \Theta \rightarrow \mathbb{R}_{+}$is logsupermodular if $\log f$ is supermodular.

To see the usefulness of log-supermodularity, assume again that the candidate expects to win the voter's vote only if $\epsilon \leq a-\theta$. The candidate believes that $\epsilon \sim F$, where $F$ is a strictly increasing, absolutely continuous distribution with density $f$. Thus the candidate wins with probability

$$
p(a, \theta)=F(a-\theta)
$$

and the transformed objective function is

$$
\log F(a-\theta)+\log u(a) .
$$

What are the comparative statics of this problem? Consider the effect of a change in the name recognition of the incumbent on the level of campaign spending by the challenger. The first derivative of the objective function with respect to $a$ is

$$
\frac{f(a-\theta)}{F(a-\theta)}+\frac{u^{\prime}(a)}{u(a)} .
$$

The level of spending $a$ will be increasing in the incumbent's name recognition if this derivative is increasing in $\theta$, which is true if $\frac{f}{F}$ is a decreasing function.

It turns out that this condition that $\frac{f}{F}$ (called the hazard rate) be decreasing is equivalent to an empirically testable claim about the distribution. In particular, this condition will hold if the density $(f)$ is logconcave. While logconcavity of the density is not a qualitatively intuitive assumption, it has two desirable properties. First, it seems a relatively innocuous assumption since most of the standard assumptions we use in formal and statistical modeling (normal, uniform, extreme value, beta, Weibull, etc.) are logconcave (Bagnoli and Bergstrom 1989). Further, one can use nonparametric methods to empirically evaluate whether or not logconcavity is present (An 1997). Thus, by using the trick of taking logs of the original objective function, we are able to show that the problem has monotone comparative statics as long as the distribution of shocks in the stochastic voting model is logconcave.

\section{Strict Conclusions}

The monotonicity theorems that we have presented only tell us that $a^{*}$ is weakly increasing in a parameter-we have not yet ruled out intervals of $\theta$ over which $a^{*}$ is constant. Sometimes it is possible to go further and show that the solution must be strictly increasing. To do this, we must strengthen the assumptions in two ways. First, we assume that $f$ is differentiable in $a$. Second, we assume that $\frac{\partial f}{\partial a}(a, \theta)$ is strictly increasing in $\theta$. Notice that the 
second condition implies that the incremental return $f(a$, $\theta)-f\left(a^{\prime}, \theta\right)$, is strictly increasing in $\theta$ as opposed to the earlier increasing differences condition, which was that it was weakly increasing in $\theta$.

Now we are ready for a strict comparative statics result. We have assumed that $f$ has strictly increasing incremental returns and is differentiable. This clearly implies that $f$ has increasing differences, so the earlier monotonicity theorem implies that $a^{*}(\theta) \geq a^{*}\left(\theta^{\prime}\right)$. We want to show that, under the stronger conditions, this inequality is strict.

Assume that $a^{*}(\theta)$ is in the interior of $A$. Since $a^{*}(\theta)$ is optimal at $\theta$, the first-order condition holds, which implies that:

$$
\frac{\partial}{\partial a} f\left(a^{*}(\theta), \theta\right)=0 .
$$

If the comparative statics are not strict, then $a^{*}\left(\theta^{\prime}\right)=$ $a^{*}(\theta)$. Thus, for the comparative statics to be weak it must be that the optimal choice under $\theta^{\prime}$ is characterized by:

$$
\frac{\partial}{\partial a} f\left(a^{*}(\theta), \theta^{\prime}\right)=0
$$

But we have assumed that $\frac{\partial f}{\partial a_{i}}(a, \theta)$ is strictly increasing in $\theta$, so both equalities cannot hold simultaneously. Since we know that the equality in equation (1) holds, it must be that $a^{*}(\theta) \neq a^{*}\left(\theta^{\prime}\right)$. Moreover, we already know from the earlier monotonicity theorem that $a^{*}(\theta) \geq a^{*}\left(\theta^{\prime}\right)$. Thus, $a^{*}(\theta)>a^{*}\left(\theta^{\prime}\right)$.

This result is formalized in the following theorem.

Theorem 3. (Edlin and Shannon (1998)) Assume $f$ is differentiable and $\partial f(a, \theta) / \partial a$ is strictly increasing in $\theta$. If $\theta>\theta^{\prime}$ and at least one of $a^{*}(\theta)$ and $a^{*}\left(\theta^{\prime}\right)$ is in the interior of $A$, then $a^{*}(\theta)>a^{*}\left(\theta^{\prime}\right)$.

We can use this result to strengthen the earlier results from the appeasement problem. Since the objective function in that problem is differentiable, we can conclude that the optimal offer is strictly decreasing at any $q$ where $x^{*}(q)$ is not 0 or 1 .

\section{Monotone Comparative Statics for Game Theoretic Problems}

Monotone comparative statics tools are useful beyond decision theory - they also can be employed in strategic settings. For this, we must extend the idea of complementarity to strategic complementarity. Informally, a game is a situation where several decision makers make choices and all of them care about the choices of the others. A game has strategic complementarities if each player wants to choose a higher action when another player increases her actions. We will start with a simple example to show how some of the decision-theoretic monotone comparative static results developed above can be used in a game-theoretic setting. We will then progress to techniques specifically suited to analyzing comparative statics of equilibria of game theoretic models.

Consider the following model of an arms race between two states. A state, $i$, has to choose a level of investment in arms, $a_{i}$. The state cares both about how well armed it is as well as how well armed its rival, $j$, is. Further, investment in arms is costly. The cost of an investment $a_{i}$ is given by $c_{i} a_{i}$. The payoff to state $i$ is given by:

$$
u_{i}\left(a_{i}, a_{j}\right)=v\left(a_{i}-a_{j}\right)-c_{i} a_{i}
$$

and to state $j$ by:

$$
u_{j}\left(a_{i}, a_{j}\right)=v\left(a_{j}-a_{i}\right)-c_{j} a_{j},
$$

where $v(\cdot)$ is increasing and concave.

An equilibrium of this game is a pair $\left(a_{i}^{*}, a_{j}^{*}\right)$ such that $a_{i}^{*}$ is a best response to $a_{j}^{*}$ and vice-versa. To do comparative statics we will take a closer look at the best response functions. For player $i$ the best response function (which determines the optimal level of $a_{i}$ ) is a function of both $a_{j}$ and $c_{i}$, which we will write $B R_{i}\left(a_{j}, c_{i}\right)$. We can use our previous results to show that $B R_{i}$ is increasing in $a_{j}$ and decreasing in $c_{i}$. To see this note first that

$$
\frac{\partial^{2} u}{\partial a_{i} \partial a_{j}}=-v^{\prime \prime}\left(a_{i}-a_{j}\right)>0,
$$

since $v$ is concave. Thus, our earlier monotonicity theorem implies that the optimal $a_{i}$ is increasing in $a_{j}$, so that $B R_{i}$ must also be increasing in $a_{j}$. Similarly,

$$
\frac{\partial^{2} u}{\partial a_{i} \partial c_{i}}=-1<0
$$

so the optimal $a_{i}$ is decreasing in $c_{i}$.

So far we have treated player i's decision problem in isolation. However, we would like to think about strategic interactions between $i$ and $j$. As we have already seen, a decrease in the cost of arms to player $i$ increases the level of arms in which player $i$ invests. Further, an increase in player is investment leads to an increase in player $j$ 's investment. Since a Nash equilibrium requires each player to choose a best response to the other's choice, this suggests that a decrease in player $i$ 's costs will increase the level of arms investment by both players.

In order to formalize this intuition we need to think more generally about the nature of equilibria in game theoretic models. A Nash equilibrium is a profile of actions $\left(a_{1}, \ldots, a_{n}\right)$ such that, for all $i, a_{i}=B R_{i}\left(\boldsymbol{a}_{-\mathbf{i}}\right)$, where 


\section{Figure 3 An Increasing Function Has at Least One Fixed Point But a Decreasing Function with Jumps Need Not}

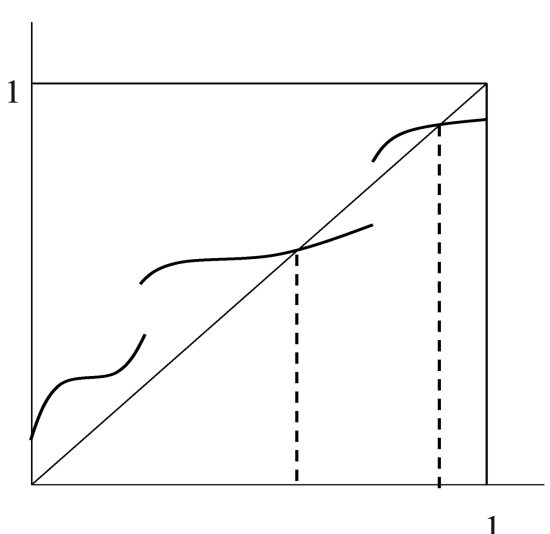

An increasing function with jumps

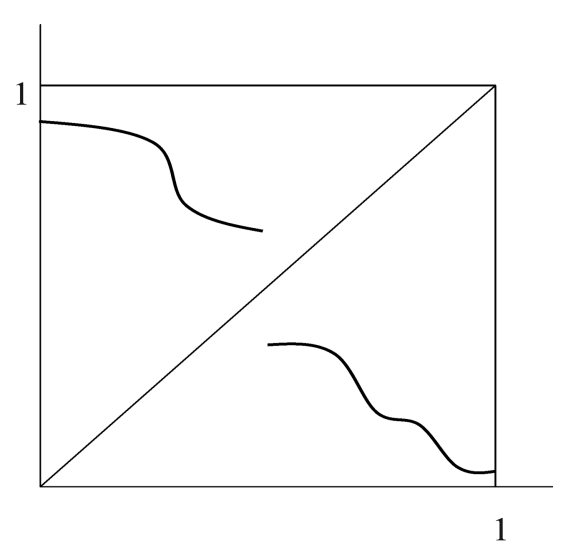

A decreasing function with jumps
$B R_{i}\left(\boldsymbol{a}_{-\mathbf{i}}\right)$ is player $i$ 's best response to the actions $\boldsymbol{a}_{-\mathbf{i}}$ of all the other players. We can write a profile of actions as a vector $a$, and say that it is a Nash equilibrium if $\mathbf{a}=\mathbf{B R}(\mathbf{a})$, where $\mathbf{B R}(\mathbf{a})=\left(B R_{1}\left(\boldsymbol{a}_{-1}\right), \ldots, B R_{n}\left(\boldsymbol{a}_{-n}\right)\right)$. An equilibrium, then, is a fixed point of the map taking the vector a to $\mathbf{B R}(\mathbf{a})$. Thus we need to extend the earlier comparative statics ideas from optimization problems to fixed-point problems.

We start by formally defining strategic complementarity.

Definition 6. A game has strategic complementarities if each player's decision problem has monotone comparative statics.

Notice that in a game with strategic complementarities the best response functions will be increasing. That is, a player's optimal choice is increasing in each other player's choice. Intuitively, it is not surprising that strategic complementarity is sufficient to insure that if something increases one player's best response function, then all the optimal actions will increase, just as occurred when the costs to one player decreased in the arms race game. This intuition reflects the following theorem. ${ }^{13}$

Theorem 4. (Tarski) Let $\mathbf{f}=\left(f_{1}, f_{2}, \ldots, f_{n}\right)$, where $f_{i}: A \times \Theta \rightarrow \mathbb{R}$. If each $f_{i}$ is increasing in a and $\theta$, then

\footnotetext{
${ }^{13}$ This result was introduced into game theory by Topkis (1979). Further results were obtained by Milgrom and Roberts $(1990,1994)$ and Vives (1990).
}

a greatest and least fixed point exist, and they are increasing in $\theta$.

The first part of the theorem (the existence of fixed points) is illustrated in Figure 3. A fixed point in these twodimensional drawings is a value of $x$ such that the value of the function (call it $f$ ) is also $x$ (that is, $x=f(x)$ ). The picture on the right demonstrates that a decreasing function does not necessarily have a fixed point - a decreasing function with jumps need not cross the 45-degree line. The picture on the left shows that an increasing function (even if it has jumps) always has a fixed point. To convince yourself of this try to draw a function (with or without jumps) from $x=0$ to $x=1$ such that it never crosses the 45 degree line (that is, $x$ never equals $f(x)$ ).

The second part of the theorem (monotone comparative statics) is illustrated in Figure 4. It is assumed that the function is increasing in the parameter. Thus, when the parameter increases from $\theta^{\prime}$ to $\theta$, the function increases pointwise. The result is that both the least and greatest fixed points (marked in the figure) increase. Notice, that, as in the figure, fixed points other than the greatest and least may decrease rather than increase in the parameter. This is not of major concern, however, since Echenique (2002) shows that if equilibrium sets are ordered this way, then a broad class of adaptive adjustment processes will converge to greater equilibria whenever a shock increases the equilibrium set.

These game-theoretic techniques can be applied to a range of substantive models of interest to political scientists. For instance, Tarski's fixed-point theorem formalizes our earlier discussion of the arms race. We showed that the best response functions in the arms race model are 

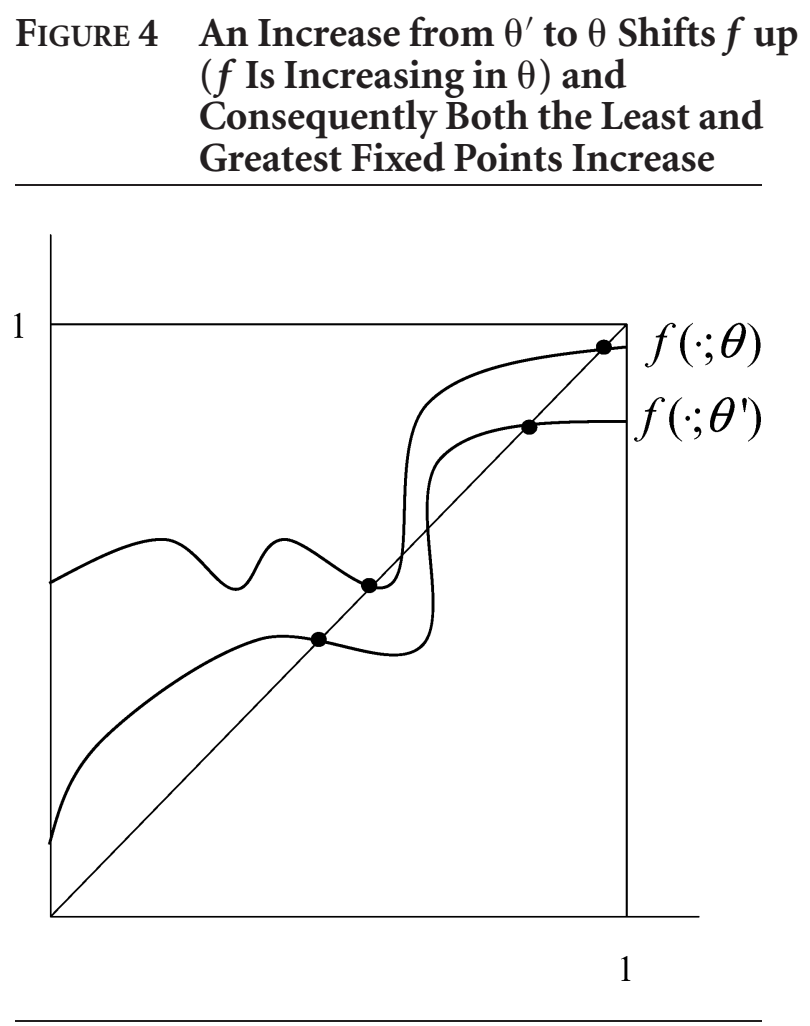

increasing in the actions (the level of weapons investment) and the parameters (the cost of weapons). Given this, Tarksi's fixed-point theorem establishes that an equilibrium exists and that it has monotone comparative statics.

\section{Comparative Statics Under Uncertainty}

Many of the most important formal theory papers of the past 20 years have featured incomplete information. Thus it is important that monotone comparative statics techniques can simplify the analysis of these models as well. From an abstract perspective, extending the results to include uncertainty is easy-the expected payoff function must satisfy the single-crossing condition. Indeed, the appeasement problem and the campaigning problem we studied in earlier sections are already problems of choice under uncertainty.

Nonetheless, it is worthwhile to examine some results that are specific to uncertainty. The standard model of choice under uncertainty is expected payoff maximization - the decision maker maximizes

$$
U(a, s)=\int u(a, \theta) f(\theta, s) d \theta,
$$

where $a$ is the choice variable and $\theta$ is a parameter that is unknown to the decision maker. The decision maker's beliefs are represented by the probability density $f(\cdot, s)$, where $s$ is a parameter indexing distributions. The optimal choice is written $a^{*}(s)$.

As always in monotone comparative statics, we want to determine when $a^{*}$ is weakly increasing in $s$. Athey (2002) exploits the special structure of expected payoff maximization to find conditions on the Bernoulli utility function $(u)$ and the probability distribution $(f)$ that together imply that the expected utility function $(U)$ is single crossing. In applications, it is often easier to check these more primitive conditions on utilities and distributions than to check directly whether the expected utility function is single crossing.

The easiest way to understand the intuition for Athey's result is to start with the special case in which the unknown parameter $\theta$ can take only two values $\bar{\theta}>\underline{\theta}$. In this case, expected utility maximization says

$$
\max _{a}(\operatorname{Pr}(\bar{\theta}) u(a, \bar{\theta})+\operatorname{Pr}(\underline{\theta}) u(a, \underline{\theta})) .
$$

Assume that the Bernoulli utility function, $u$, is single crossing in $a$ and $\theta$. If $\theta$ is known, then this is a problem without uncertainty, and Theorem 1 tells us that the optimal $a$ is weakly increasing in $\theta$ (that is, $a^{*}(\bar{\theta}) \geq a^{*}(\underline{\theta})$ ). In the case with uncertainty over $\theta$, it is intuitive that $a^{*}$ should be weakly increasing in $\operatorname{Pr}(\bar{\theta})$. That is, if the optimal action is weakly increasing in the parameter in the complete information case, then the optimal action should be weakly increasing in the probability that the parameter takes on the high value in the incomplete information case.

It is easy to prove this intuitive conclusion if we strengthen the assumption that $u$ is single crossing and instead assume that it has increasing differences. Let $p=\operatorname{Pr}(\bar{\theta})$, and write the expected payoff as

$$
U(a, p)=p u(a, \bar{\theta})+(1-p) u(a, \underline{\theta}) .
$$

We want to show that the optimal choice $\left(a^{*}(p)\right)$ is weakly increasing in $p$. To do this, we need only confirm that $U(a$, $p$ ) has increasing differences. Differentiating with respect to $p$ yields

$$
\frac{\partial}{\partial p} U(a, p)=u(a, \bar{\theta})-u(a, \underline{\theta}) .
$$

This derivative is increasing in $a$, since $u$ has increasing differences. Thus $U$ has increasing differences and the optimal $a$ is weakly increasing in $p$.

As demonstrated in this simple example, in order to study monotone comparative statics in incomplete information environments, we need a way to order probability distributions. In the example with just two values of $\theta$, 
this is easy-one distribution is greater than a second if and only if it assigns higher probability to the larger value of $\theta$. In the two value case, then, we can say that a distribution that assigns probability $p$ to $\bar{\theta}$ is greater than another distribution that assigns probability $p^{\prime}$ to $\bar{\theta}$ if $p>p^{\prime}$.

It turns out to be useful to rewrite this condition in a different way. We will say that a distribution $\left(f\left(\cdot, s_{1}\right)\right)$ that assigns probability $p$ to the higher parameter value is greater than a distribution $\left(f\left(\cdot, s_{2}\right)\right)$ that assigns probability $p^{\prime}$ to the higher parameter value if

$$
\frac{p}{1-p}>\frac{p^{\prime}}{1-p^{\prime}}
$$

When there are only two values, this is just a somewhat odd way of saying $p>p^{\prime}$. The important fact, as we will see, is that this form of the condition is the "right" one for generalization to cases with more than two possible parameter values.

If we extend this ratio inequality to hold for all pairs $\theta_{1}, \theta_{2} \in \Theta$, even when there are more than two values of $\theta$, then we say that the $s_{1}$ distribution likelihood ratio dominates the $s_{2}$ distribution. This is a strong notion of "larger" for random variables. ${ }^{14}$

Definition 7. A distribution $f\left(\cdot, s_{1}\right)$ monotone likelihood ratio dominates or is larger in the likelihood ratio order than a distribution $f\left(\cdot, s_{2}\right)$, if for all $\theta_{1}>\theta_{2}, \theta_{1}$, $\theta_{2} \in \Theta$,

$$
\frac{f\left(\theta_{1}, s_{1}\right)}{f\left(\theta_{2}, s_{1}\right)} \geq \frac{f\left(\theta_{1}, s_{2}\right)}{f\left(\theta_{2}, s_{2}\right)}
$$

We will also use the following, closely related definition.

Definition 8. A family of distributions satisfies the monotone likelihood ratio property if it is indexed by parameters, $s$, that can be ordered such that $f\left(\cdot, s^{\prime}\right)$ monotone likelihood ratio dominates $f\left(\cdot, s^{\prime \prime}\right)$, for all $s^{\prime}>s^{\prime \prime}$.

This definition simply says that if distributions can be rank ordered in terms of monotone likelihood ratio dominance, then they are said to satisfy the monotone likelihood ratio property.

Athey's theorem shows that the monotone likelihood ratio order is the notion of larger needed to extend single crossing to the case of uncertainty with more than two possible parameter values. We state two of her results, one for one-dimensional problems and one that applies to multidimensional problems.

\footnotetext{
${ }^{14}$ The appendices to Krishna (2002) provide a useful summary of the large literature on orders for random variables.
}

\section{Theorem 5 (Athey).}

1. Assume that $A$ is one-dimensional. If $u$, is single crossing in $a$ and $\theta$, and $f$ satisfies the monotone likelihood ratio property, then $U(a, s)=\int u(a, \theta) f(\theta, s) d \theta$ is single crossing in a and sand, therefore, $a^{*}(\cdot)$ is weakly increasing in $s$.

2. If $u$ is either supermodular or log-supermodular, and $f$ satisfies the monotone likelihood ratio property, then $a^{*}$ is weakly increasing in s. $^{15}$

We already know how to check the conditions on $u$. However, in order for this theorem to be useful, we must also be able to check whether the distribution satisfies the monotone likelihood ratio property.

Recall from the definition that the likelihood ratio order requires

$$
\frac{f\left(\theta_{1}, s_{1}\right)}{f\left(\theta_{2}, s_{1}\right)} \geq \frac{f\left(\theta_{1}, s_{2}\right)}{f\left(\theta_{2}, s_{2}\right)} .
$$

We can take logs of both sides and rewrite the condition as

$\log f\left(\bar{\theta}, s_{1}\right)-\log f\left(\underline{\theta}, s_{1}\right) \geq \log f\left(\bar{\theta}, s_{2}\right)-\log f\left(\underline{\theta}, s_{2}\right)$.

Thus a family of distributions has the monotone likelihood ratio property if and only if the density is log-supermodular. Thus the monotone likelihood ratio property can be verified by computing the cross partial derivative of $\log f$. Hence, Theorem 5 implies that the expected utility function $(U)$ will be single crossing if the Bernoulli payoff function $(u)$ is single crossing and the density function is log-supermodular.

We have described how to extend the single-crossing property, and thereby monotone comparative statics, to problems involving uncertainty. However, the question remains as to whether there is any intuition underlying the assumption that the family of densities is ordered by the likelihood ratio order. Milgrom (1981) shows that this ordering on distributions is particularly natural in problems where the distributions are derived from Bayesian updating.

To see why, assume that the decision maker has a prior distribution $f$ on $\Theta$ and that she observes a signal with likelihoods $p(s \mid \theta)$. Using Bayes's rule, her posterior distribution is then

\footnotetext{
${ }^{15}$ Van Zandt and Vives (2003) show that a weaker condition, that the $s$ index the distributions in the sense of first-order stochastic dominance, is sufficient if the Bernoulli utility is supermodular. We emphasize Athey's result because the likelihood ratio ordering is easier to check in applications and because it is general enough to apply to the kind of utility functions that arise in probabilistic voting model and to the use of monotone comparative statics in the median voter theorem.
} 


$$
f(\theta \mid s)=\frac{p(s \mid \theta) f(\theta)}{\int p\left(s \mid \theta^{\prime}\right) f\left(\theta^{\prime}\right) d \theta} .
$$

The problem will have monotone comparative statics if the posterior distribution conditioned on a given signal monotone likelihood ratio dominates the posterior distribution conditioned on a lower signal.

To see how this is related to the model's primitives, rewrite Bayes's rule in odds form:

$$
\frac{f(\theta \mid s)}{f\left(\theta^{\prime} \mid s\right)}=\frac{p(s \mid \theta)}{p\left(s \mid \theta^{\prime}\right)} \frac{f(\theta)}{f\left(\theta^{\prime}\right)} .
$$

From this expression it is clear that the posteriors will be ordered in the likelihood ratio order if and only if the likelihood ratio $\frac{p(s \mid \theta)}{p\left(s \mid \theta^{\prime}\right)}$ is increasing in $s$. Substantively, this will be true if, for any two values of the parameter $(\theta)$, higher values of the signal make the higher parameter value relatively more likely. This condition is satisfied for many standard parametric forms of the likelihood (normal, etc.).

Likelihoods that satisfy this condition also arise quite naturally in models of measurement error. Assume that the signal is a noisy observation of the true parameter value, $s=\theta+\epsilon$, where $\epsilon$ is a mean zero random variable independent of $\theta$ distributed according to a logconcave density, $f$. The likelihood ratio is $\frac{p(s \mid \theta)}{p\left(s \mid \theta^{\prime}\right)}=\frac{f(s-\theta)}{f\left(s-\theta^{\prime}\right)}$. Since $f$ is logconcave, the likelihood ratio is increasing in $s$.

It will be instructive to see how these techniques can be applied in the appeasement problem we have already examined. Assume that the relative military strength of the satisfied state is $q(\theta)$, where $\theta$ is $D$ 's strength and $q$ is decreasing. Further assume that $S$ knows its own strength, but must rely on intelligence reports to assess $D$ 's strength. The intelligence service produces a signal, $s=\theta+\epsilon$, where $\epsilon$ is independent of $\theta$ and has a logconcave density, $f$. Due to the argument in the previous paragraph, $S^{\prime}$ s posterior beliefs $(f(\theta \mid s))$ satisfy the monotone likelihood ratio property. The expected utility function is given by

$$
\begin{aligned}
U_{S} & =\mathbb{E}[p(x)(1-x)+(1-p(x)) q(\theta)] \\
& =\int(p(x)(1-x)+(1-p(x)) q(\theta)) f(\theta \mid s) d \theta
\end{aligned}
$$

Our earlier treatment of the appeasement problem, without uncertainty, showed that

$$
u_{S}=p(x)(1-x)+(1-p(x)) q(\theta)
$$

is single crossing in $x$ and $\theta$. Given that $f(\theta \mid s)$ satisfies the monotone likelihood ratio property, Theorem 5 implies that $U_{S}$ is single crossing, and therefore the optimal offer $\left(x^{*}(s)\right)$ is weakly increasing in the signal $(s)$ - the higher the signal of D's military strength, the larger the offer of appeasement.

\section{Implications for Empirical Work}

We have demonstrated a variety of ways in which monotone comparative static techniques aid formal theorists in building, solving, and deriving results from models. We have also discussed how qualitative empirical research can help to evaluate whether the assumptions necessary for monotone comparative statics hold. We conclude by discussing several ways in which monotone comparative statics have benefits for researchers who want to combine formal models and quantitative empirical research. ${ }^{16}$

There are several approaches to testing the empirical implications of theoretical models. An increasingly popular technique is to derive a complete likelihood function from a formal model augmented only by random noise. This structural modeling approach has been applied in each of the empirical subfields. A few examples include Diermeier, Eraslan, and Merlo's (2003) work on government formation in parliamentary systems, Smith's (1999) and Signorino's (1999) estimations of a variety of models of crisis bargaining in international relations, and Gowrisankaran, Mitchell, and Moro's (2003) estimate of the incumbency advantage in the United States Senate based on a formal model of electoral selection. The primary benefit of this approach is that it allows the researcher to fully estimate the structure of an equilibrium model. This provides a strong test of the theory and facilitates counterfactual simulations which are useful for prediction and policy evaluation.

As Signorino and Yilmaz (2003) point out, the validity of this approach to estimation depends on the functional forms implied by the formal model accurately reflecting the true data-generating process. Often, however, formal theorists think of their models as partial treatments, intended to isolate particular mechanisms of interest, but failing to capture the full complexity of the political situation being studied. In these situations, it may not be feasible to do structural estimation. Nonetheless, even if we cannot test the full specification of the model, we want to be able to test some of the predictions of the model, to determine whether the basic causal mechanisms the model identifies are important. When the monotone comparative statics approach applies, it facilitates this more limited type of testing of theoretical models by providing some assurance of the robustness of comparative static predictions even with an incompletely specified model.

When we model a decision problem by assuming that a decision maker maximizes $f(a, \theta)$, we intend to highlight the role of $\theta$ in the determination of $a$. We typically do

\footnotetext{
${ }^{16}$ For an example of an empirical application where the single crossing condition emerges, see King and Wand (2004).
} 
not intend to suggest that there are no other important determinants of $a$. That is, we think the true data-generating process results from the maximization of $f(a, \theta)+g(a)$, for some arbitrary function $g$. Ideally, our results would be robust to such arbitrary perturbations that do not directly affect the particular relationship we are studying. Such robustness does not hold for all decision problems, but it does hold if $f$ has increasing differences. In that case, $f(a, \theta)+g(a)$ satisfies the single crossing condition for all $g$. Thus increasing differences justifies drawing comparative statics conclusions from partially specified models. What is important for comparative statics is not fully specifying the problem, but only correctly specifying the parts of the problem where the variables of interest interact.

By comparing the right-hand panels of Figures 1 and 2 it is easy to see why increasing differences gives us this kind of robustness but single crossing on its own does not. No matter how one shifts the graph of the incremental return in Figure 2, it will remain single crossing. However, a shift of the incremental return in Figure 1 could easily cause the graph to cross zero more than once. More formally, the incremental return in the perturbed problem is

$$
f(a, \theta)-f\left(a^{\prime}, \theta\right)+g(a)-g\left(a^{\prime}\right) .
$$

For any value of $g(a)-g\left(a^{\prime}\right)$, this incremental return will be increasing, and hence single crossing, in $\theta$ if $f$ has increasing differences.

Monotone comparative statics approaches provide some robustness against incorrect specification, and thus yield the type of prediction that can be tested even when we are not confident enough in our theoretical model to test its full specification. Because the comparative static predictions do not depend on precisely specifying the functional form, it is natural to test these limited claims nonparametrically, without making any assumptions except those necessary for monotonicity. ${ }^{17}$ This can be done, for example, by using rank tests, either on the raw data or on the residuals from regressions of the variables of interest on a vector of control variables (Rosenbaum 2002).

For example, recall that in our model of the appeasement problem the level of expenditures on military buildup $(z)$ and the offer of concession $(x)$ were negatively correlated. Since, as discussed above, monotone comparative statics are invariant to monotone transformations of the domain, we can test this prediction of the model with only ordinal information, using Spearman's rank correlation test. Hence, when we are concerned that misspecifications of our theoretical model might limit the validity of structural estimation, but there are monotone compar-

\footnotetext{
${ }^{17}$ For a discussion of the use of nonparametric methods in political science, see Beck and Jackman (1998).
}

ative statics, we can suggest an alternative approach that still allows us to test the empirical implications of theoretical models with confidence in the robustness of our conclusions.

It is important to note that monotone comparative statics do not provide robustness against arbitrary specification errors in standard linear models. Signorino and Yilmaz (2003) point out that omitted nonlinearity leads to omitted variable bias, and they show that simple strategic models of deterrence imply nonlinearities that make estimators like logit and probit inconsistent. They identify one case where the standard linear-index estimators may be appropriate: the left-hand side variable must be unconditionally monotonic in the regressors. Since omitted variable bias typically leads to bias in all of the coefficients, one needs this unconditional monotonicity in all of the regressors. Monotone comparative statics typically do not yield this strong result. In particular, the results described in this article are ceteris paribus results. In empirical applications, this means that the researcher needs control variables to isolate the effects described by the theory. But monotone comparative statics says nothing about unconditional monotonicity of the left-hand-side variable in these control variables.

Thus, in our view, monotone comparative statics and associated nonparametric methods should be viewed as complementary to structural approaches. Monotone comparative statics, for instance, can play an important role in the approach advocated by Signorino and Yilmaz (2003) in which the researcher iterates between reduced form and structural models. By providing predictions that are robust to specification error, monotone comparative statics contribute by allowing for nonparametric testing that can then lead to revised theoretical models that approach a true model of the data generating process, leading ultimately to a model that can be structurally estimated.

\section{References}

An, Mark Yuying. 1997. "Log-Concave Probability Distributions: Theory and Statistical Testing.” Duke University Department of Economics Working Paper No. 95-03.

Athey, Susan. 2002. "Monotone Comparative Statics Under Uncertainty." Quarterly Journal of Economics CXVII(1):187223.

Bagnoli, Mark, and Ted Bergstrom. 1989. "Log-Concave Probability and Its Applications.” Unpublished paper. University of Michigan.

Baron, David. 1994. "Electoral Competition with Informed and Uninformed Voters." American Political Science Review 88(1):33-47. 
Beck, Nathaniel, and Simon Jackman. 1998. "Beyond Linearity by Default: Generalized Additive Models.” American Journal of Political Science 42(2):596-627.

Bueno de Mesquita, Bruce, James D. Morrow, Randolph M. Siverson, and Alastair Smith. 1999. "An Institutional Explanation of the Democratic Peace." American Political Science Review 93(4):791-808.

Cameron, Charles, and Rebecca Morton. 2002. Formal Theory Meets Data. In Political Science: The State of the Discipline, Vol. III, ed. Ira Katznelson and Helen Milner. New York: W.W. Norton, pp. 784-804.

Diermeier, Daniel, Hulya Eraslan, and Antonio Merlo. 2003. “A Structural Model of Government Formation.” Econometrica 71(1):27-70.

Echenique, Federico. 2002. "Comparative Statics by Adaptive Dynamics and the Correspondence Principle." Econometrica 70(2):833-44.

Edlin, Aaron S., and Chris Shannon. 1998. "Strict Monotonicity in Comparative Statics." Journal of Economic Theory 81(1):201-19.

Erikson, Robert S., and Thomas R. Palfrey. 2000. "Equilibria in Campaign Spending Games: Theory and Data." American Political Science Review 94(3):595-609.

Fearon, James D. 1995. “Rationalist Explanations for War.” International Organization 49(3):379-414.

Gans, Joshua S., and Michael Smart. 1996. "Majority Voting with Single-Crossing Preferences." Journal of Public Economics 59(2):219-37.

Gowrisankaran, Gautam, Matthew F. Mitchell, and Andrea Moro. 2003. "Why Do Incumbent Senators Win? Evidence from a Dynamic Selection Model." Unpublished paper, Washington University.

Grossman, Gene M., and Elhanan Helpman. 1996. "Electoral Competition and Special Interest Politics." Review of Economic Studies 63(2):265-86.

King, Gary, and Jonathan Wand. 2004. "Comparing Incomparable Survey Responses: New Tools for Anchoring Vignettes.” Unpublished Paper, Harvard University.
Krishna, Vijay. 2002. Auction Theory. San Diego: Academic Press.

Milgrom, Paul, 1981. "Good News and Bad News: Representation Theorems and Applications." Bell Journal of Economics 12(2):380-91.

Milgrom, Paul, and Chris Shannon. 1994. "Monotone Comparative Statics." Econometrica 62(1):157-80.

Milgrom, Paul, and John Roberts. 1990. "Rationalizability, Learning, and Equilibrium in Games with Strategic Complementarities." Econometrica 58(6):1255-77.

Milgrom, Paul, and John Roberts. 1994. "Comparing Equilibria." American Economic Review 83(3):441-59.

Powell, Robert. 2002. Game Theory, International Relations Theory, and the Hobbesian Stylization. In Political Science: The State of the Discipline, Vol. III, ed. Ira Katznelson and Helen Milner. New York: W.W. Norton, pp. 755-83.

Rosenbaum, Paul. 2002. "Covariance Adjustment in Experiments and Observational Studies." Statistical Science 17(3):286-327.

Signorino, Curtis S. 1999. "Stategic Interaction and the Statistical Analysis of International Conflict." American Political Science Review 93(2):279-98.

Signorino, Curtis S., and Kuzey Yilmaz. 2003. "Strategic Misspecification in Regression Models." American Journal of Political Science 47(3):551-66.

Smith, Alastair. 1999. "Testing Theories of Strategic Choice: The Example of Crisis Escalation." American Journal of Political Science 43(14):1254-83.

Topkis, Donald. 1978. "Minimizing a Submodular Function on a Lattice." Operations Research 26(2):305-21.

Topkis, Donald. 1979. "Equilibrium Points in Nonzero-Sum nPlayer Submodular Games." SIAM Journal of Control and Optimization 17(6):773-87.

Van Zandt, Timothy, and Xavier Vives. 2003. "Monotone Equilibria in Bayesian Games of Strategic Complementarities." Unpublished Manuscript. INSEAD.

Vives, Xavier. 1990. "Nash Equilibrium with Strategic Complementarities." Journal of Mathematical Economics 19(3):30521. 